\title{
Linx
}

Revue des linguistes de l'université Paris X Nanterre

9 | 1997

Émile Benveniste. Vingt ans après

\section{Actif et moyen dans le verbe}

\section{Françoise Bader}

\section{(2) OpenEdition \\ Journals}

Édition électronique

URL : http://journals.openedition.org/linx/966

DOI : $10.4000 /$ linx.966

ISSN : 2118-9692

\section{Éditeur}

Presses universitaires de Paris Nanterre

\section{Édition imprimée}

Date de publication : 1 avril 1997

Pagination : 41-59

ISSN : 0246-8743

\section{Référence électronique}

Françoise Bader, «Actif et moyen dans le verbe », Linx [En ligne], 9 | 1997, mis en ligne le 03 juillet

2012, consulté le 02 mai 2019. URL : http://journals.openedition.org/linx/966 ; DOI : 10.4000/linx.966 


\title{
Actif et moyen dans le verbe
}

\author{
Françoise Bader
}

1. Benveniste et la diathèse, catégorie fondamentale du verbe indo-européen

2. Supplétisme verbo-pronominal: 2.1. préverbes en fonction d'espace et d'altérité; particules modales; préverbes aspectuels; 2.2. personne et nombre ; 2.3. désinences de diathèse (type $\left.{ }^{*}-t\right)$ et finales temporelles (type $\left.{ }^{*}-t-i\right)$

3. Diathèse et temps : 3.1. indifférence au temps des désinences de diathèse ; 3.2. paradigmes hors temps : 3.2.1. injonctif ; 3.2.2. parfait

4. Benveniste et le sémantisme d'Actif et Moyen dans le verbe : 4.1. définition de B. ; 4.2. méthode et critiques : 4.2.1. matériaux ; 4.2.2. dissymétrie des Activa et des Media tantum; 4.2.3. problème de la chronologie relative du moyen et de l'actif

5. Exemple d'antériorité flexionnelle du moyen sur l'actif : I sg.

6. Caractéristiques morphologiques moyennes de la forme fondamentale (degré zéro, flexion moyenne)

7. Polyvalence des diathèses sémantiques de la forme moyenne fondamentale : 7.1. diathèses statique (interne) et dynamiques (interne et externe); 7.2. ${ }^{*} d e h_{3^{-}},{ }^{*} h_{1} e r-;{ }^{*} h_{1} e u-\left({ }^{*} h_{1} w-e s-\right.$ et $\left.{ }^{*} h_{1} w-e b h-\right)$, *kel- (*kl-u- et $\left.{ }^{*} k l_{\circ}-h_{1^{-}}\right)$;

7.4. oppositions lexicales des diathèses sémantiques

8. Expansion des catégories verbales à partir du moyen fondamental : 8.1. remarques sur la théorie benvenistienne de la racine; 8.2. procédés d'expansion (différenciations radicales; intégration par affixes) et tableaux

9. Chronologie relative des "thèmes temporels" : 9.1. parfait et présent du type $\kappa \tilde{\varepsilon \imath}-\mu \alpha \imath$; 9.2. aoriste ; 9.3. actif : 9.3.1. actif morphologique ; 9.3.2. actif sémantique

10. Transfert du moyen à l'actif: 10.1. perfectum latin du type dedī; 10.2. présents hittites en $-h i$; 10.3. présents thématiques; 10.4. extension de l'actif aux présents thématiques; 10.5 . présents à degré zéro de moyen et flexion active (type dat) ; 10.6. variétés de paradigmes mixtes 
Françoise Bader

11. Transfert de l'actif au temps ( ${ }^{*} e s-m i$, etc.)

12. Scission de l'ancien moyen en moyen sémantique et moyen résiduel : 12.1.

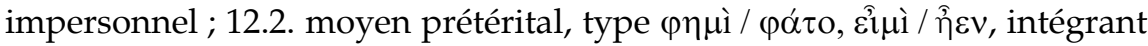
au temps l'opposition morphologique entre Actif et Moyen dans le verbe.

\section{Actif et moyen dans le verbe}

1. Dans "Actif et moyen dans le verbe" (1950), E. Benveniste proclame le caractère primordial de la diathèse : "Nous avons bien affaire à une catégorie fondamentale, et qui se lie, dans le verbe indo-européen, aux autres déterminations morphologiques. Ce qui caractérise en propre le verbe indoeuropéen est qu'il ne porte référence qu'au sujet, non à l'objet. A la différence $d u$ verbe des langues caucasiennes ou amérindiennes par exemple, celui-ci n'inclut pas d'indice signalant le terme (ou l'objet) du procès... Tout est présenté et ordonné par rapport au sujet. Mais les catégories verbales qui se conjoignent dans les désinences ne sont pas toutes également spécifiques: la personne se marque aussi dans le pronom; le nombre, dans le pronom et dans le nom. Il reste donc le mode, le temps, et, par-dessus tout la 'voix', qui est la diathèse fondamentale du sujet dans le verbe" (p.169). B. proclame, par ailleurs, le caractère primordial, à l'intérieur de la diathèse, de l'opposition actif/moyen ("toute forme verbale finie relève nécessairement de l'une ou de l'autre diathèses, et même certaines des formes nominales $d u$ verbe (infinitifs, participes) y sont également soumises", p.169), après avoir mis à part le passif (p.168) -dont je dirai qu'il est une spécialisation du moyen en regard d'un actif transitif, dans une conjugaison à double diathèse flexionnelle-. Et il définit ainsi l'objet de son étude (p.169) : "quel sens attribuerons-nous à la distinction entre actif et moyen? C'est le problème que nous examinerons sommairement". Et mon propre propos est d'essayer d'aller au-delà, tout en étant fortement inspirée par les lignes puissantes de mon maître.

Que la diathèse soit bien la catégorie fondamentale du verbe indoeuropéen, caractérisée par l'opposition moyen/actif ressort de l'existence de deux séries de désinences (Watkins, 1969, 66) :

$\begin{array}{lllll} & \text { Sg. I } & \text { II } & \text { III } & \text { Plur. III } \\ \text { moyen } & *-h_{2} e & *-t h_{2} e & -e / 0 & * \ldots . . . \\ \text { actif } & *-m & *-s & *-t & *-n t\end{array}$

(compte non tenu des restructurations de désinences moyennes consécutives au développement de l'actif, et qui se font par infixation de la désinence active, comme à la III sg. ${ }^{*}-t-o$ sur ${ }^{*}-o$, à la I sg. ${ }^{*}-m-h_{2} e i>{ }^{*}$ mai sur ${ }^{*}-h_{2} e-i-$ à la II sg. ${ }^{*}-s-t h_{2} e>$ gr. $(\tau i \theta \eta) \sigma \theta a$, etc.).

2. Se pose alors le problème du rapport à la diathèse des autres catégories, "temps, mode, personne, nombre", et plus loin "nombre, mode, temps, voix", dit 
B. (p.169), qui oublie l'aspect. La réponse à ce problème est donnée par le supplétisme verbo-pronominal. Des particules pronominales (au nombre desquelles les préverbes) ont en effet doté le verbe de leurs catégories (personne-altérité, espace-temps, qui relient le locuteur à sa situation objective dans le monde, et auxquelles s'ajoute celle de l'incertain, qui relève de la subjectivité : indéfinitude, interrogation, modalités diverses, notamment l'éventualité, le désir, etc.).

2.1. Des préverbes pronominaux ont en effet doté le verbe des catégories d'espace et d'altérité, ainsi du thème *ke de lat. (hi-)c, osq. ce-(bnust) "uenerit", lat. con-(uenio) "venir avec". Des particules pronominales du type de gr. $\kappa \epsilon$ ont constitué l'expression la plus ancienne du mode, la seule que connaissent les langues anatoliennes, hors de l'impératif, qui s'oppose aux "modes de l'imaginaire", subjonctif et optatif, dont les formations spécifiques dénoncent d'ailleurs le caractère récent : ou bien ce sont d'anciens présents, devenus des éventuels par la formation d'un nouveau présent qui, forme axiale de la conjugaison temporelle, se renouvelle (ainsi $\imath^{\prime} \omega$, cf. hitt. iy-a-hha, à côté de $\epsilon^{\hat{\imath}} \mu \mathrm{u}$ ); ou bien ce sont des formes dérivées, en rapport avec des présents et des prétérits, en ${ }^{*}-\bar{a}-$,

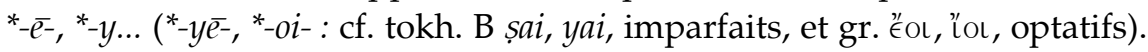
En sens chronologique inverse, les préverbes aspectuels (lat. cōn-ficiō, -fécîl), se sont développés après la forme marquée sous le rapport de l'aspect, le parfait, pour doter la conjugaison temporelle dans tous ses thèmes de la valeur d'accompli de ce dernier, et pour en diversifier les aspects. Pour ce qui est du supplétisme verbo-pronominal, je m'attarderai davantage à la personne (à laquelle est conjointe le nombre) et surtout au temps.

2.2. Selon B., dont l'enseignement est important sur ce point (p.174), "la diathèse s'associe aux marques de la personne et du nombre pour caractériser la désinence verbale. On a donc, réunies en un même élément, un ensemble de trois références qui, chacune à sa manière, situent le sujet relativement au procès et dont le groupement définit ce qu'on pourrait appeler le champ positionnel du sujet : la personne, suivant que le sujet entre dans la relation 'je-tu' ou qu'il est 'non-personne' (dans la terminologie usuelle '3ème personne'); le nombre, suivant qu'il est individuel ou plural ; la diathèse enfin, selon qu'il est extérieur ou intérieur au procès". Or, l'étymologie des désinences verbales peut être fonction de la "Structure des relations de personne dans le verbe" (B., 1946) : à la III p., les désinences évoquent au sg. les formations nominales de noms d'agent thématiques et en ${ }^{*}-t$ - (III moy. ${ }^{*}-e / o$, act. $\left.{ }^{*}-t\right)$, au pluriel (moy. ${ }^{*}-r . .$. ; act. ${ }^{*}-n t$ ) l'hétéroclisie nominale (sur laquelle voir B., 1935, 3-22), et le caractère parfois pluralisant de ${ }^{*} n-$; mais aux I et II les désinences verbales peuvent relever du supplétisme verbo-pronominal : l'association de la personne et du nombre, ce dernier de nature différente dans le pronom et dans le nom, donne en faveur de l'origine pronominale de ces désinences un argument plus fort que l'homophonie de telle désinence et de tel pronom personnel comme ${ }^{*}-m$ et ${ }^{*}-m i$; mais cette homophonie peut 
Françoise Bader

par ailleurs jouer un rôle dans la mesure où le même thème peut apparaître au singulier et au pluriel, ce qui renvoie à l'indifférence au nombre des anaphoriques (mise en valeur par B., 1933), ainsi *-m I sg. et ${ }^{*}-m_{\%}^{e}$ I plur., auxquelles s'agglutinent, de plus, des particules différentielles à l'égard de la diathèse : ainsi - $m u-s$ et $-\mu \epsilon-v$ à l'actif, $-\mu \epsilon-\theta a$ au moyen ; etc.

2.3. Le supplétisme verbo-pronominal se fait beaucoup plus clair pour le temps (auquel B. a aussi consacré des réflexions fécondes, s'agissant du français : 1959) : une étude sur Actif et Moyen dans le verbe doit en effet distinguer des désinences de diathèse proprement dites (type $\left.{ }^{*}-t\right)$ les finales temporelles (type ${ }^{*}-t-i$ ).

L'expression du temps a en effet été obtenue dans la flexion du verbe i.e. par des particules pronominales temporalisantes, le plus souvent postposées aux désinences de diathèse; dans un cas particulier, la particule est préposée à la forme verbale fléchie : c'est celui de l'augment, dans une aire dialectale continue comprenant l'indo-iranien, l'arménien, le phrygien, le grec. Et on posera p.ex. à partir de la III sg. * ${ }^{*} t e h_{2}-t$ active :

${ }^{*}{ }^{*} t e h_{2}-t+{ }^{*}-i$ : lat. stat, skr. stháti, subjonctif, ancien présent ;

${ }^{*} e-+{ }^{*}{ }^{*} t e h_{2}-t$ : gr. ${ }^{*} \sigma \tau \eta(\tau)$, skr. ásthât, aoriste.

L'origine du système où le présent et le prétérit sont distingués par des particules doit être recherchée au moyen : ainsi de *ēe- "être assis", cf. $\hat{\eta} \mu a$, etc. (§ 8.1.) en hittite (où -ta vaut $-\theta a$ à la II et -To à la III) :

$\begin{array}{llll} & \text { sg. I } & \text { II } & \text { III } \\ \text { prés. } & \text { eshaha-ri } & \text { esta-ri } & \text { esa-ri } \\ \text { prét. } & \text { esha-t }(i) & \text { esta- } t & \text { esa-t }(i), \text { esta-t. }\end{array}$

Deux cas se présentent, selon que chacun des temps est marqué par une particule différentielle, comme dans cet exemple (et cf. skr. sthát-i/á-sthät), ou que seul l'est le présent, forme marquée du système temporel : кєîTo$\iota / \kappa \in \hat{\imath}$ то. La particule temporalisante peut varier dans ses formes et

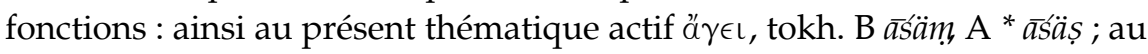
moyen *sekw-e-to $+-i,-r(i)>$ skr. sácate, lat. sequitur; à côté de $\imath \epsilon$, qui n'est marqué comme prétérit que par son opposition à $\epsilon \hat{\imath} \sigma \mathrm{l}$, le grec a à la III du prétérit de "aller", $\iota \epsilon(\nu)$, et avec augment, $\eta_{\imath} \epsilon(\nu)$, $\eta_{i} \epsilon L$, cf. (red-)iei-t, § 10.4. ; facultative, la "nasale éphelcystique" est fixée à la III sg. "il était" $\hat{\eta} \in-\nu<{ }^{*} e_{-}$ $h_{1} s-e-n$ (devenue homophone de la III plur. $\left.\hat{\eta} \in v(\tau)<{ }^{*} e-h_{1} s-e n t\right)$. Ces variations, consécutives à l'asémantisme des pronoms, ne font qu'illustrer le fait général selon lequel un même thème pronominal peut assumer plusieurs fonctions, et corrélativement une fonction donnée peut avoir plusieurs signifiants pronominaux (ainsi ${ }^{*} k{ }^{w} i$ - est relatif en fonction syntaxique d'articulation, et interrogatif-indéfini en fonction catégorielle $\mathrm{d}^{\prime}$ incertitude ; le relatif est tantôt ${ }^{*} k^{w} i$-, tantôt ${ }^{*}$-yo- ; etc.). Naturellement, le choix de ces particules peut être fonction de conditions articulatoires : ${ }^{*}-t$ ne s'adjoint que ${ }^{*}-i$; mais on a ${ }^{*}-e-i,{ }^{*}-e-n,{ }^{*}-e-s . . .$, comme à côté de $\imath \imath \nu$, $-i \in \iota$, dans tokh. B yam, A yäs "il va". 
3. Cette analyse segmentale $\left({ }^{*}-e\right.$ moyen, ${ }^{*}-t$ actif $+{ }^{*}-i$, etc., temporalisant) est un fait qui donne à penser que le temps n'est pas la catégorie la plus ancienne dans le verbe i.e. Il y en a d'autres.

3.1. L'un est un corollaire de la distinction entre finales temporelles et désinences de diathèse : celles-ci sont indifférentes au temps, et peuvent se trouver aux deux temps qui forment le plus ancien noyau de la conjugaison temporelle, ainsi hitt. -ha: -ha-ri et -ha-t, § 2.3.

3.2. Mais surtout deux paradigmes, qui s'opposent sous le rapport de l'origine de leurs désinences et de leur aspect, restent pour ainsi dire à l'abri du temps au développement duquel ils sont antérieurs : l'injonctif et le parfait.

3.2.1. L'injonctif, sujet choisi par l'un des cinq auteurs des Etrennes....Benveniste (Renou, 1928, citant un autre de ces auteurs : J.Kurylowicz, Injonctif et subjonctif dans les Gāthās, Lwów, 1927), est défini formellement comme forme sans augment et à désinences secondaires (donc actives), c.à.d. comme dépourvu des marqueurs pronominaux temporalisants, ce qui explique qu'il soit "zeitstufenlos", selon Hoffmann (1967, 265-266), qui en donne la définition fonctionnelle suivante (278-79) : "So bezeichnet also eine Injunktivform die Noeme lexikalische Bedeutung (einschliesslich Aktionsart), Aspekt, Person (einschliesslich Numerus), Diathese und Erwähnung; mit anderen Worten: Eine Injunktivform erwähnt eine Verbalhandlung in ihrem Aspekt mit Angabe und Diathese". Hoffmann a d'autant plus raison de considérer que ce paradigme n'est pas "primitif" (p.35) que, d'une part, il appartient au système de l'actif, postérieur à celui du moyen de manière générale ( $\$ 5.12$ ), et que, d'autre part, ses aspects sont ceux des thèmes auxquels il appartient, présent et aoriste, aspects respectivement duratif et ponctuel, qui se sont développés par opposition à l'accompli, que dénote le parfait, lui-même d'apparition plus ancienne, dans l'expansion de la conjugaison, que le présent (\$ 9.1.) et l'aoriste (§ 9.2.).

3.2.2. Or le parfait, forme marquée du point de vue de l'aspect, est lié au moyen sémantiquement (B., 1949), et morphologiquement: il n'a de flexion que moyenne (type gr. $-\alpha,-\theta a,-\epsilon$ ), si bien que lorsqu'un parfait actif se formera dans une conjugaison à double diathèse, il aura, à l'origine en distribution allophonique, ou bien une flexion moyenne (type $\lambda \epsilon ́ \lambda$ oเta, qui conjoint les deux marques des plus anciens parfaits, vocalisme ${ }^{*}-0$ - et redoublement, cf. § 8.2.), ou bien des désinences zéro en supplétisme avec des désinences moyennes (skr. papráu, paprátha, papráu). Le parfait aurait donc mérité de figurer parmi les Media tantum de B.

4. Si celui-ci, qui a consacré d'importantes études au parfait (1949 ; 1952 ; $1959 b ; 1962,41-65 ; 1970 b$ ) n'en parle pas, et du coup oublie l'aspect dans l'énumération des catégories verbales, c'est en raison de la méthode qu'il met en oeuvre pour définir le contenu sémantique d"'Actif et Moyen dans le verbe". 
Françoise Bader

4.1. Ses conclusions seront les suivantes : "dans l'actif, les verbes dénotent un procès qui s'accomplit à partir du sujet et hors de lui. Dans le moyen, qui est la diathèse à définir par opposition, le verbe indique un procès dont le sujet est le siège; le sujet est intérieur au procès...dont il est l'agent", p.172... "ces oppositions... reviennent toujours en définitive à situer des positions du sujet vis-à-vis $d u$ procès, selon qu'il y est extérieur ou intérieur, et à le qualifier en tant qu'agent, selon qu'il s'effectue, dans l'actif, ou qu'il effectue, en s'affectant, dans le moyen", p.173 ; et "si l'on convient de substituer aux termes 'actif' et 'moyen' les notions de 'diathèse externe' et de 'diathèse interne', cette catégorie retrouve plus facilement sa nécessité dans le groupe de celles que porte la forme verbale", p.174.

4.2. Au point de départ de cette recherche sémantique, se trouve la définition que donne Pāninini de présents à double diathèse, comme yájati "il sacrifie (pour un autre, en tant que prêtre)" et yájate "il sacrifie (pour soi, en tant qu'offrant)". Et c'est pourquoi B. n'a opéré qu'avec des présents. Ayant discuté du parti à tirer de cette interprétation (Problèmes, 170), il a eu l'idée, féconde, de partir, au contraire, des Activa tantum et Media tantum (p.171-172).

4.2.1. La première remarque qu'appellent ces deux listes est que $B$. n'opère qu'avec des présents du sanskrit, grec, latin, en apparence de manière assez scolaire, mais en réalité, je crois, parce qu'il part de l'interprétation de Pānini, que le verbe grec s'accorde bien avec le verbe indien (notamment pour les innovations), et que le latin est souvent associé au grec pour des raisons plus culturelles que linguistiques. Cela étonne, il est vrai, de la part de l'auteur de Hittite et Indo-Européen (1962); et il faut peut-être souligner ici que ce livre est de douze ans postérieur à Actif et Moyen, et que la plus ancienne des sept études de morphologie verbale qui s'y trouvent ("Présents dénominatifs en hittite") date de 1955 ; cela revient en tout cas à négliger des faits aussi importants pour l'histoire de la diathèse que la conjugaison hittite en $-h i$, ou que les finales en $-r(i) \mathrm{du}$ hittite, du tokharien (sur lequel B. a écrit un important article : 1936), de l'italique ou du celtique, finales à distinguer de la désinence en *...r... de III plur. moyenne, et procédant d'une recaractérisation du moyen consécutive au transfert du moyen à l'actif $(\$ 10)$, du type *-o-r à côté de *$\bar{o}$ (qui recouvre formellement le hittite $-a-h h a$, resté moyen).

4.2.2. En second lieu, B. ne prend pas conscience de la dissymétrie qui oppose aux Activa tantum les Media tantum. Ces derniers ont une flexion unique, que leur structure soit athématique (skr. sé-te, gr. $\kappa \in \hat{\imath}-\mathrm{Tol}$ ) ou thématique (skr. s'áy-a-te). Mais il existe deux variétés de flexions actives: l'une dite athématique, (du type de skr. váti, gr. önoı), l'autre dite thématique, ellemême à deux variantes, des types skr. sárpati (à désinence identique à

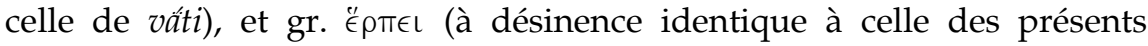
hittites en $-i$, etc. : $\$ 10.2$.). Or entre ces deux flexions thématiques actives, il n'existe aucune opposition sémantique : leur coexistence est seulement le fruit de la lente différenciation d'un actif et d'un moyen plus ancien que lui. 
4.2.3. Et B. n'a pas posé le problème de la chronologie relative de l'actif et du moyen, auquel je me consacrerai jusqu'à la fin de cette étude. Je distinguerai à la suite de P.Flobert (1984), entre voix morphologique et diathèses sémantiques. De la "voix morphologique" relève l'appareil formel de la diathèse (flexions, degrés radicaux); de la diathèse sémantique, les oppositions entre diathèse statique et diathèse(s) dynamique(s), cf. $\$ 7$; toute l'histoire de la diathèse est déterminée par l'adéquation à la diathèse sémantique dynamique d'une voix morphologique active, qui ne s'est imposée que lentement, après d'autres essais de différenciations des diathèses sémantiques, élargissements radicaux différentiels (\$ 7.3.), transfert du moyen à l'actif (§ 10), et qui a fini elle-même par être dominée par le temps (§ 11).

5. De l'antériorité du moyen sur l'actif témoigne l'histoire des désinences, beaucoup plus compliquée, parce que beaucoup plus longue, pour les désinences moyennes que pour les désinences actives. D'une part en effet, le développement de la flexion active reste inachevé, puisqu'il n'est aucune des finales temporelles issues d'elles qui soit pandialectale (la plus largement répandue étant celle de la III plur., * $\left.{ }^{*}(\%) n t i\right)$, et qu'il y a des langues (périphériques) qui ignorent complètement * ${ }^{*} m i$, comme le latin, qui n'a de désinence d'origine active que dans s-um, et le tokharien B, dont l'unique présent à désinence de même origine est yam "je vais". D'autre part, alors qu'une désinence comme ${ }_{-}{ }_{-} \% m$ ne pose d'autre problème que son emploi comme désinence de présent et de prétérit (cf.

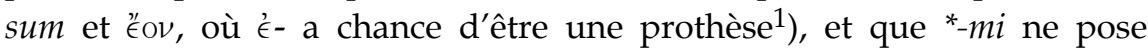
$\mathrm{d}$ 'autre problème que la dominance du temps sur la diathèse dans "être" (§ 11), les désinences d'origine moyenne ont subi de nombreuses réfections formelles et ont été affectées à des emplois divers, qu'on illustrera sommairement pour ${ }^{*}-h_{2} e$, I sg. moyenne ; elle a donné :

-ha de présent et prétérit moyens en hittite (\$ 2.3.) ;

- $a$ au parfait du type gr. oîsa, skr. véda ;

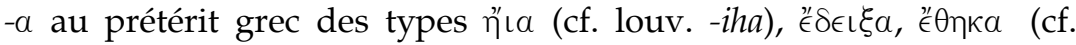
$\S 12.2.) ;{ }^{*}-\bar{o}$ au présent thématique actif, formellement superposable au moyen hitt. - $a$-hha (à structure thématique) : ${ }^{*}-0-h_{2} e>{ }^{*}-\bar{o} a$ (avec ${ }^{*}-\bar{o}$ - par chute de ${ }^{*} h_{2}$ et allongement compensatoire) $>-\bar{o}$ (résultant d'un transfert du moyen à l'actif : § 10) ;

${ }^{*}-\bar{O} r$ (hitt. -a-hha-ri), par l'innovation archaïque qu'est la recaractérisation moyenne du présent consécutive au transfert de ${ }^{*}-\bar{O}$ à l'actif ;

avec particule ${ }^{*}-i\left({ }^{*}-h_{2} e-i\right)$ :

-é védique de présent moyen ;

-hi hittite, de présent actif, résultant d'un transfert du moyen à l'actif ;

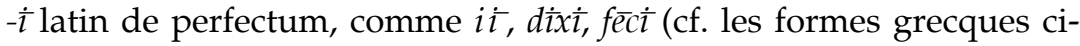

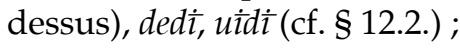

\footnotetext{
${ }^{1}$ Mais voir, maintenant, les exemples italiques de esom.
} 
Françoise Bader

*-mai de présent grec et prétérit tokharien $\mathrm{B}$, né d'une hypercaractérisation de moyen par infixation de la désinence active (§ 1).

6. De l'antériorité du moyen sur l'actif, voici d'autres témoignages.

L'un est donné par les caractéristiques morphologiques de la forme fondamentale, forme verbale unique fléchie que fournissent les racines les plus anciennes : flexion moyenne ; degré zéro généralisé. Cette forme, qui est au point de départ de l'expansion de la conjugaison (\$ 8), survit dans tokh. B ste < ${ }^{*} s_{2} h_{2}-0$, seul "présent" démuni de la particule temporalisante *-n, précisément parce que, fournissant la copule, il est hors temps ; mais elle est plus souvent intégrée au temps, soit par une particule temporalisante comme présent (type skr. vidé, iyé), qui peut être thématisé (skr. tudáti), soit comme moyen prétérital (louv. -iha, etc.).

7. Or cette forme, morphologiquement moyenne, est polyvalente à l'égard des diathèses sémantiques.

7.1. Celles-ci sont les diathèses statique (et "interne" dans la terminologie de B., en tant qu'elle "ne porte référence qu'au sujet, non à l'objet", cf. § 1), et dynamiques de deux sortes, l'une également "interne", l'autre "externe", qui illustre le fait suivant mis en lumière par B. (p.172-73): "supposons qu'un verbe typiquement moyen tel que gr. коциа̃тal "il dort" soit doté secondairement d'une forme active. Il en résultera, dans la relation du sujet au procès, un changement tel que le sujet, devenant extérieur au procès, en sera l'agent, et que le procès n'ayant plus le sujet pour lieu, sera transféré sur un terme qui en deviendra objet. Le moyen se convertira en transitif. C'est ce qui se

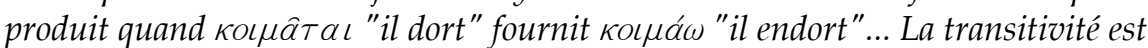
le produit nécessaire de cette conversion du moyen à l'actif. Ainsi se constituent à partir du moyen des actifs qu'on dénomme transitifs ou causatifs ou factitifs".

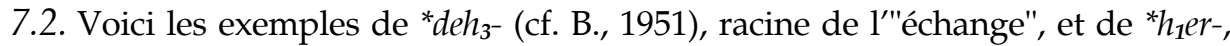
racine la plus ancienne pour la position "debout", suppléée par *st-eh ${ }^{-}$, une fois qu'elle s'est appliquée au "mouvement" auquel est destiné le fait de "se lever" (sauf dans le cas où l'on monte la garde, auquel s'applique la forme hittite de la col. 1)

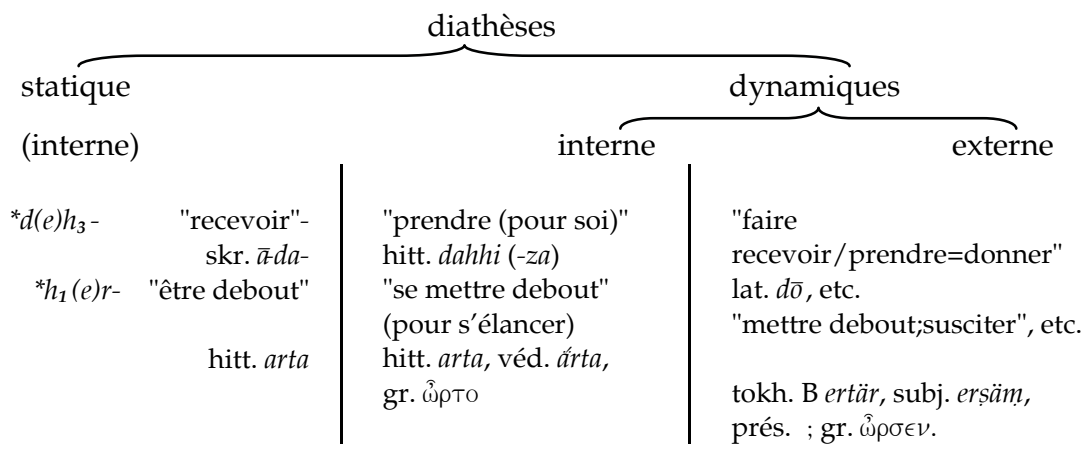


Les signifiants de diathèse sémantique sont ici véd. $\overline{a-}$, préverbe qui prend le relais du moyen sémantique, hitt. -za particule réflexive (employée notamment pour l'homme qui "prend" femme), ${ }^{*}-s-$, morphème de diathèse dynamique au présent et au prétérit ("aoriste sigmatique"); pour le vocalisme ${ }^{*}-0$ - de $\hat{\rho} \rho$ To, ertär, cf. § 9.1.

7.3. Voici d'autres exemples de phénomènes semblables, montrant de plus que les diathèses sémantiques ne sont pas nécessairement au nombre de trois, et que les oppositions de diathèses ont pu se faire par des thèmes différentiellement élargis. Les racines choisies sont celle de la "vêture", ${ }^{*} h_{1}$ eu-, et du "bruit", *kel- :

diathèse statique diathèse dynamique

${ }^{*} h_{1}(e) u-\quad$ "s'habiller = recevoir du tissage": "faire du tissage $:$ habiller" : lat. (ind, ex-)ū̄ skr. v-áya-ti, ó-tum.

Deux points sont à noter. D'une part, le statique $-u \bar{o}$ conserve le degré zéro du moyen ancien, avec une thématisation elle-même issue du moyen (§ 10.3.), et les formes dynamiques offrent des marques différentielles: v-áya- a le même degré radical de même origine, et une formation suffixale, -áya-, dynamique (et le plus souvent causative) ; ó-tum, infinitif, relève de l'actif morphologique, par son vocalisme plein, probablement de timbre ${ }^{*} e$. D'autre part, toujours, avec le degré zéro du moyen, les deux formes se sont opposées par des élargissements radicaux :

${ }^{*} h_{1} w$-es- statique pour la vêture / ${ }^{*} h_{1} w$-ebh- dynamique pour le tissage.

A l'intérieur du premier s'est développée l'opposition "s'habiller", ëvvvua

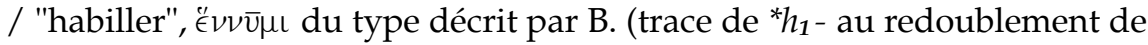
skr. vō̄asāná-: * $h_{1} w e-h_{1} w$-es-).

Dans un domaine comme celui du son, l'opposition entre les diathèses

est : statique dynamique

"l'homme reçoit le bruit : il entend" "le tonnerre, l'eau, etc., fait du bruit"

(verbe corporel de perception): $\quad$ (verbe physique d'émission) :

gr. к`úw, skr. śṛnuté; etc.

tokh. AB käln-, lat. clangō, etc. ; et

"l'homme appelle, invoque", etc., si

l'émission est produite par la voix :

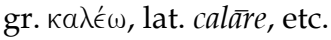

Avec toujours le degré zéro du moyen, les verbes corporels de perception et d'émission de la voix s'opposent comme

$$
\text { *kl-u- } \quad * k l-h_{1^{-}} \text {. }
$$

Des morphèmes peuvent en outre spécifier le caractère statique ou dynamique des formes: le ${ }^{*}-\bar{e}-$ statif de lat. clueō prend le relais de l'ancien moyen statique ; le -n- de käln- est un morphème de présent dynamique, le -s- de hitt. kalles- (présent), gr. Ł̇אá $\epsilon \in \sigma \alpha$ (aoriste), un morphème de présent et

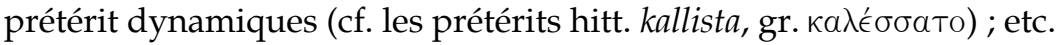

7.4. Dans les synchronies de l'époque historique, une opposition du type

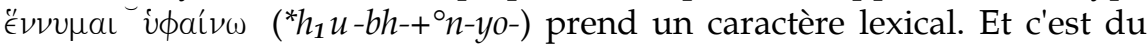


Françoise Bader

lexique que relève l'opposition, en hittite et en grec, de *kei- statique et *dheh $h_{1}$ - dynamique.

8. L'expansion de la conjugaison, où l'actif morphologique va entrer en scène, témoigne aussi de l'antériorité du moyen sur l'actif.

8.1. Je prendrai ici deux exemples de racines élargies, ${ }^{*} w e i-d$ - et ${ }^{*} s t-e h_{2^{-}}$, d'une structure dégagée lumineusement par B. (1935, 147-173), et à laquelle n'échappe aucune racine ancienne. Je donnerai de cela trois ordres d'arguments. D'une part, les laryngales initiales ne sont pas rétablies par B. (cf. 1935, 149) pour les besoins théoriques de la racine "trilittère" (terme que B. doit avoir emprunté à la grammaire sémitique, où la plupart des racines ont trois consonnes, non deux comme le degré zéro des racines non élargies) : ainsi, l'augment long de $\ddot{n} \in \nu$, et allongement compensatoire) de ${ }^{*} e^{--+}{ }^{*} h_{1} s_{-},{ }^{*} h_{1} y_{-}$; le même traitement (qui est celui de la forme redoublée vā̃asāná-, § 7.3.) explique aussi la longue de *⿳亠丷厂s- "être assis" (§ 2.3.), intégration à l'aide du redoublement (cf. $\S$ 8.2.) de la forme moyenne fondamentale ${ }^{*} h_{1} s-e$ (celle qui figure dans $\eta \models(v)$, consécutive à l'emploi de * $h_{1}$ es- pour "être", par un supplétisme tiré $\mathrm{d}^{\prime}$ un verbe de position corporelle qu'illustre, de la racine de la position debout, tokh. B ste (§ 8.2.), ou fr. il était (sur "être", cf. B., 1960, et sur ses rapports avec La phrase nominale, 1950). D'autre part, la théorie de l'infixe nasal $(1935,159-163)$ permet de rendre à certaines racines leur structure benvenistienne ; en voici trois exemples : pour ${ }^{*} s t-e h_{2}-(1935,158$, sans

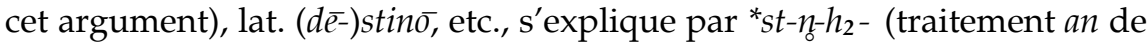
*n devant * $\left.h_{2}\right)$; pour skr. bhánati "parler", gr. фaí $\nu \omega$ "briller", une analyse

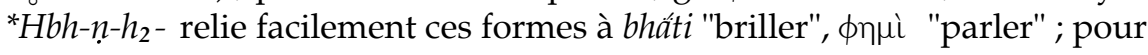

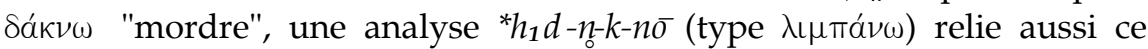
présent au type lat. $e d \bar{\sigma}$ "manger" (laryngale donnée par $\bar{e} d \dot{\imath}<{ }^{*} h_{1} e-h_{1} d-$ ); "mordre" d'où est issu "manger" subsiste au sens métaphorique "faire mal" dans óbúrn "douleur", etc., et au sens propre au présent renouvelé (par l'infixe, dynamique) $\delta a ́ k \nu \omega$, et dans le nom de la "dent" (lat. dēns, etc.), sur lequel B. a émis des vues sémantiquement justes (1931), tout en le retirant à "manger", dont il n'a pas vu qu'il procédait de "mordre". Enfin (last, not least), le fait que 'quantité de "racines" sont en réalité des thèmes II' $(1935,154)$, reçoit appui de formes comme ${ }^{*} h_{1} w-e s-,{ }^{*} h_{1} w-e b h-(\$ 7.3$.$) ,$ auxquelles on ajoutera maintenant ${ }^{*} h_{1} w-e r-(g)->(\dot{\epsilon}) \dot{\epsilon} \rho \gamma \omega$, etc. (avec une prothèse témoignant de l'existence d'une laryngale initiale), racine de l'ouverture-fermeture" d'un habitat conçu métaphoriquement à l'image de la vêture (avec un élargissement alors sémantiquement différentiel).

8.2. C'est à partir de la forme moyenne fondamentale qu'ont été bâtis les divers "thèmes temporels", selon deux procédés (dont la coexistence explique celle de deux types de parfaits, comme lat. lìquì, véd. riricé, ou de présents,

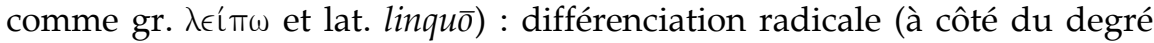
zéro du moyen fondamental, *-o-/zéro au parfait, *-e- généralisé au présent 


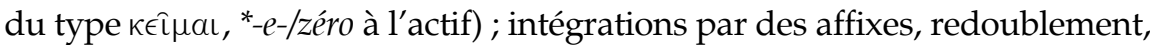
infixe, suffixes. Ici encore, les formes issues du moyen dont elles ont la flexion, parfaits du type *wóide et présents du type *kéi-to, sont plus nombreuses que celles qui sont nées de l'actif, témoignant d'une histoire plus longue. Je mêle dans mon tableau les deux familles en lesquelles *wei- $d$ - s'est scindé en sanskrit : "savoir", sens issu du parfait ; "trouver", issu de "voir", comme terme de la quête visuelle. J'écris en majuscules ce qui relève de la forme moyenne fondamentale, ainsi VID-Á-ti et VI-n-D-Á-ti; ces derniers conservent sur ce qui est devenu structure thématique l'accent qui portait la désinence de *wid-é (l'accent ne frappant pas en principe un degré radical zéro); skr. vidé résulte de l'intégration au présent de l'ancien moyen par particule temporalisante ; démuni d'une telle particule, tokh. B ste "il est" (copule), est resté en dehors de cette évolution (\$ 6) :

\begin{tabular}{|c|c|c|}
\hline & $\begin{array}{l}\text { différenciation } \\
\text { radicale }\end{array}$ & $\begin{array}{l}\text { intégration } \\
\text { par affixes }\end{array}$ \\
\hline *wid-é : & 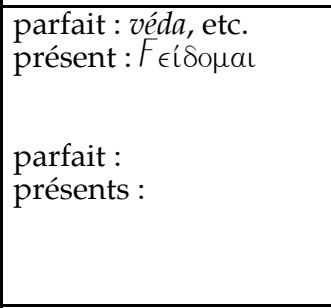 & 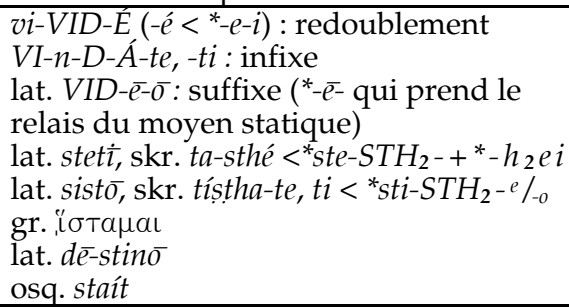 \\
\hline & 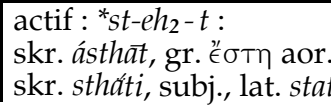 & 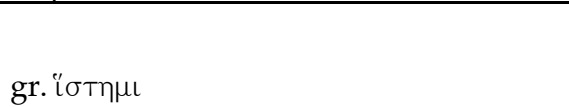 \\
\hline
\end{tabular}

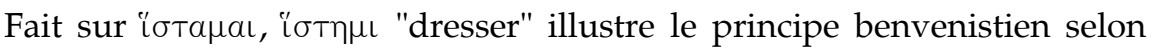
lequel, quand il est fait sur un moyen, un actif est factitif (\$ 7.1.) : les présents redoublés qui se correspondent sémantiquement et morphologiquement en sanskrit et grec sont tissthate et iotatal (avec traitement de ${ }^{*} s t h_{2}-$ consécutif à la restructuration de la désinence moyenne ${ }^{*}-e$ ( (cf. B ste) en *-to). L'ancienneté du degré zéro (du moyen fondamental) ressort de la généralisation de th en sanskrit, qui ne s'explique qu'à partir de ${ }^{*} s h_{2}-$. Dernière remarque morphologique (last, not least), * st-eh $_{2}-t$ montre que, quand un actif est fait sur le moyen à double degré zéro d'un radical élargi, il prend le thème II : c'est pourquoi les aoristes "radicaux athématiques" ont ce vocalisme quand ils sont actifs (type ásthät), non quand ils sont moyens (type $\pi \lambda \eta \hat{T}$, avatar de la forme moyenne fondamentale * ${ }_{0} l-h_{1}-t o$, devenue prétéritale, cf. $§ 12.2$.).

9. L'expansion de la conjugaison pose des problèmes de chronologie relative pour le parfait et le présent, l'aoriste, l'actif.

9.1. Du parfait et du présent, tous deux issus du moyen, le premier est le plus ancien, non seulement parce que sa flexion a été plus largement à l'abri des réfections que le présent $(-\alpha,-\theta \alpha,-\epsilon$, mais $-\mu a$, etc.), mais surtout parce 
Françoise Bader

que, hors temps (§ 3.2.2.), le parfait a été souvent intégré à celui-ci, soit comme prétérit (lat. uìd "j'ai vu", non "je sais"), soit comme présent, avec une flexion moyenne (oĩ $\mu a \mathrm{l}$ ), parfois transférée à l'actif (hitt. sakhi, § 10.2.), ou même active (v.sl. vëmŭ). En pratique, tout vocalisme *-o- dénonce un présent comme venant d'un parfait ; un cas notable est celui des formes en *h $h_{1}$ or- (cf. § 7.2.) ; la valeur aspectuelle de "s'élancer" comme accomplissement de "se mettre debout" ("s'élancer pour s'être mis debout") amène *her- à ne plus être qu'un verbe de mouvement, sa fonction de verbe de position corporelle, alors tombée en déshérence, étant relayée par ${ }^{*}{ }^{*} t e h_{2}{ }^{-}: \mathrm{d}^{\prime}$ où

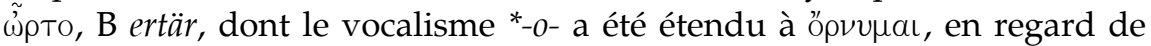
rnuté, et aux formes sigmatiques (ici de diathèse externe) $\tilde{\omega} p \sigma \in \nu, B$ erșäm.

Si le parfait est plus ancien que le présent autonome (j'entends par là celui qui a un procédé de formation spécifique, autre que l'intégration par particules), on se demandera si la catégorie de l'aspect (dont le parfait est la forme marquée) n'est pas antérieure à celle du temps (on songe ici au verbe sémitique où l'aspect est primordial, au contraire du temps). S'il en était ainsi, les catégories du verbe i.e. se seraient développées dans l'ordre : diathèse - aspect - temps - mode (de toute façon la dernière née de ces catégories, cf. § 2.1.).

9.2. Troisième thème de la conjugaison d'une langue comme le grec, avec le parfait et le présent, l'aoriste pose, lui, le problème de savoir s'il relève d'une catégorie. Si oui, c'est de l'actif, par opposition au parfait et au présent (par opposition auxquels il s'est développé comme prétérit d'aspect ponctuel); mais la réponse doit être nuancée en fonction des variétés flexionnelles d'aoristes.

D'une part, deux d'entre elles sont en rapport avec le moyen : les aoristes affixés comme $\ddot{\epsilon} \delta \in \iota \xi \alpha, \ddot{\epsilon} \theta \eta \kappa \alpha$ ont une flexion de moyen prétérital (§ 12.2.) antérieure à la flexion par désinences dites secondaires pour le prétérit (employées p.ex. à l'imparfait latin); et les aoristes radicaux athématiques moyens viennent d'une intégration au prétérit de la forme moyenne fondamentale, soit que comme $̈$ є́ Оото ils fonctionnent comme aoristes moyens d'un paradigme à double diathèse, soit que comme фáto (dont la valeur flotte entre celle d'aoriste et d'imparfait), ils n'aient pas de sémantisme particulièrement moyen, puisque фáTo sert de prétérit à $\phi \eta \mu i$, dans une conjugaison résiduelle comportant un présent et un prétérit, du type hitt. es-zi / es-ta (\$ 12.2.). Mais, d'autre part, l'aoriste, dans sa variété "radicale athématique active", est fortement relié à l'actif, dont il est une intégration temporelle (type ásthât mal représenté dans l'ensemble des langues indo-européennes). En troisième lieu, entre actif et moyen se situe la variété "radicale thématique", du type $\epsilon \hat{\delta} \delta o v$ (de "voir"), à fonction active dans la conjugaison, et à morphologie mixte : le radical y est au degré zéro du moyen (et à ce titre $F i \delta \epsilon$ peut être un avatar du moyen fondamental), mais la flexion en est mixte : comme le montre l'exemple le plus ancien, $\epsilon \hat{\imath} \delta o v$ / $\hat{i} \delta \epsilon(v)$, skr. ávidam / ávidat (et cf. arm. egit), la I y est en *-om, active 
(développée probablement sur le modèle de la III plur. en *-ont, cf. lat. sum et $s$-unt, etc.) ; et la III y montre les mêmes variétés que les présents "thématiques" (§ 10.4.) *-e-+-n, particule temporalisante, en grec où la finale de présent thématique est $-\epsilon-\mathrm{*} ;{ }^{*}-e-+-t$ en arménien et en indoiranien, où la finale de présent thématique est en ${ }^{*}-e-t i$; ce paradigme mixte existe au présent tokharien, ainsi, avec également un degré zéro originaire du moyen dans B yam / yam, A yäm / yäs de "aller".

9.3. S'agissant de l'actif, deux problèmes se posent, relatifs l'un à l'actif morphologique, l'autre à l'actif sémantique.

9.3.1. L'apparition du premier me paraît postérieure à celle du parfait, parce que la flexion de celui-ci lui est toujours demeurée étrangère. Mais elle doit être antérieure au présent du type $\kappa \in \hat{\imath} \mu a \mathrm{l}$, pour deux raisons: ce présent semble formé à une époque où les alternances ont cessé de jouer comme procédé de différenciation catégorielle, parce qu'il n'en restait plus de disponible; et une flexion - $\mu a$, - - or témoigne de l'intrusion de la flexion active. Mais le développement de l'actif n'a pas précédé d'assez longtemps celui de ces présents pour que cesse de jouer, dans ces derniers, l'ambivalence des diathèses statique ( $\kappa \in \hat{\imath} \mu a \iota)$ et dynamique ("̈тона ) du moyen fondamental ; l'actif morphologique sera le terme de l'évolution de deux des présents les plus anciens, "être" et "aller" (cf. §11).

9.3.2. Cet actif morphologique a été précédé d'autres procédés d'adéquation de formes différentielles aux diathèses sémantiques aptes à donner des actifs sémantiques, ce qui élimine la singularité que serait un système de diathèse où un moyen ne serait opposé à aucun actif : certains relèvent du lexique (§ 7.4.), comme l'emploi d'élargissements radicaux différentiels (§ 7.3.), d'autres de la morphologie, comme l'emploi d'affixes spécialisés pour la diathèse statique (ainsi ${ }^{*}-\bar{e}-$ ), ou pour les diathèses dynamiques (ainsi, l'infixe, employé dans des formes dont les plus anciennes ont le double degré zéro de l'ancien moyen, comme linquō, avant de prendre le thème II de l'actif fait sur le moyen, comme skr. rinákti), etc. : cf. § 7.2. ; 7.3. pour certains d'entre eux. Un autre procédé, afférent à la flexion, est le transfert du moyen à l'actif, dont je retiendrai quatre manifestations: le perfectum latin ; les présents en -hi du hittite; les présents thématiques des autres langues; le type lat. dat. Sauf le perfectum latin, tous ces types témoignent d'une rencontre du moyen transféré à l'actif, en sa flexion et/ou son degré radical, et de l'actif morphologique, dont la porte d'entrée semble être la 3ème du pluriel, dans les présents hittites en -hi et les présents thématiques.

10.1. Au type lat. stetì, dedì, intégré à l'actif, répondent en effet en sanskrit

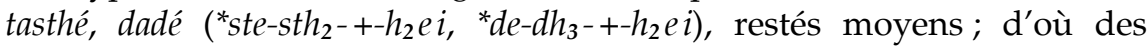
restructurations inverses dans le développement de la conjugaison à double diathèse pour lat. datus sum au médio-passif, mais skr. dadáu, tastháu à l'actif ; la flexion active étant complètement étrangère au parfait 
Françoise Bader

indo-européen, ces derniers ont des désinences zéro aux I et III sg. (en regard de la II en -tha) ; leurs correspondants grecs actifs ont une flexion moyenne grâce à l'introduction d'un morphème consonantique au

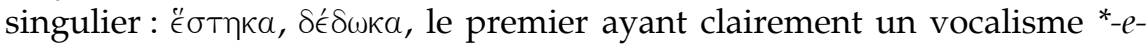
d'actif, et non *-o- de parfait, ce qui, par récurrence, doit être aussi le cas des autres exemples.

10.2. Comparés au perfectum latin, les présents hittites en - $h i$ appellent trois remarques. L'une est que la flexion en est au singulier identique à celle de ce perfectum: $-h i,-t i,-i$, cf. $-\dot{i},-t i \bar{i},-e i-t$ (à côté de $-e-d)<{ }^{*}-h_{2} e,{ }^{*}-t h_{2} e,{ }^{*}-e+$ la particule * $i$ (et cf. < véd. -é de présents restés moyens, à la I et à la III du type bruvé à côté de bruté) ; mais elle en diffère à la III plur., où le latin a des désinences en ${ }^{*}-r$ - d'origine moyenne, comme le védique (tasthúr), mais le hittite $-n z i<{ }^{*}-n t i$, qui montre que la porte d'entrée de l'adéquation à la diathèse sémantique de la voix morphologique a été cette personne (cf., au parfait grec, la III plur. en - $\bar{\alpha} \sigma \iota$ à côté du sg. $-\alpha,-\theta \alpha,-\epsilon)$. En second lieu, alors que le vocalisme du parfait redoublé est anciennement le vocalisme zéro, comme dans dedì, etc., parce qu'il résulte de l'intégration de la forme moyenne fondamentale qui a ce vocalisme, celui des présents hittites en -hi montre que ceux-ci ont intégré non seulement ce moyen, mais des parfaits, et sont devenus donc perfecto-présents: si arhi est ambigu, uhhi relève du premier type, et sakhi "je sais" (racine de lat. sc-i-i-re, lequel a le degré zéro de l'ancien moyen, intégré au présent par le suffixe $-\dot{i}-)$ du second ; les formes à vocalisme ${ }^{*}-e-\mathrm{y}$ sont encore exceptionnelles : nehhi, de la racine *nei- (sur laquelle voir Benveniste, 1962, 33-40) paraît plus récent que le moyen ney-a-hha-ri. Enfin ces présents ont une structure tantôt athématique, comme celle du perfectum latin, tantôt thématique, qui nous mène aux présents thématiques (cf. sagg-a-hhi à côté de sak-hi).

10.3. Pour comprendre la thématisation, on partira de gr. $-\epsilon \iota /-\epsilon$-Tal (réfection de $-\epsilon-T O L):-\epsilon l$ est identique à hitt. $-i$; et c'est le transfert à l'actif de cette finale moyenne qui a entraîné la recaractérisation du moyen en *-e-to, seule forme thématique pandialectale, où se conjoignent deux désinences, comme plus tard dans lat. $-r-u n t$, skr. $-\bar{a}-m i$, qui témoignent, eux, d'une recaractérisation active consécutive au transfert du moyen à l'actif.

L'extension de la thématisation aux autres personnes vient de ce qu'elle est le point de rencontre de la diathèse et du temps, dont la dialectique commande toute l'histoire du verbe indo-européen : pour la diathèse, moyen $\mathrm{d}$ 'où elle est originaire (ce qui explique le tropisme moyen des formes thématiques); actif, par transfert du moyen à la diathèse dynamique, antérieur au développement complet de l'actif morphologique, et double diathèse, du type yájati / yájate, née dans les présents thématiques ; pour le temps, la thématisation, propre au présent, apparaît comme le procédé unificateur de tous les présents antérieurs à ceux du type * statt- $i$, *éi-ti (qui se trouve au terme d'une évolution dont le point de départ est un moyen fondamental, cf. $\S 11$, et ne fait donc partie des Activa tantum qu'au bout 
de son histoire), en d'autres termes de tous les présents non actifs, encore liés au moyen et aux formes issues de lui par leurs vocalismes, sorte de carte d'identité de leur origine: présents résultant d'une intégration par particule du moyen originel, à vocalisme zéro et, consécutivement, accent anciennement sur la désinence (type skr. tudáti); ou du parfait à vocalisme ${ }^{*}-0$ - (hitt. sagg-a-hhi); présents nés du type le plus ancien de présents autonomes, à vocalisme *-e, comme le montrent bien, de *kei-,

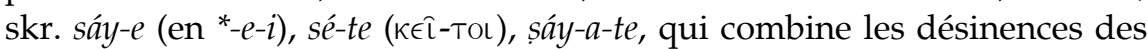
deux formes précédentes en ayant fait passer la première, ${ }^{*}-e-$, au rang de structure thématique; $c^{\prime}$ est ce vocalisme ${ }^{*}-e$ - qui est le plus vivant aux présents radicaux thématiques, "immobiles" du point de vue des alternances comme leur modèle medium tantum.

10.4. La flexion de ces présents est plus ou moins conservatrice à l'égard des désinences d'origine moyenne au singulier : hitt. $-h i,-t i,-i$ est entièrement $\mathrm{d}$ 'origine moyenne. L'actif commence à s'y développer en grec, où à côté de $-\omega$ (cf. hitt. $-a-h h a$, resté moyen), $-\epsilon L$ (cf. hitt. $-i$, devenu actif), la II, en $-\epsilon L-$, s'explique par l'adjonction de l'actif * ${ }^{*} s$ non à ${ }^{*} e$, mais à ${ }^{*}-e i$, avec sa particule; cette structure remarquable est aussi celle des prétérits,

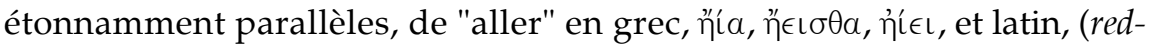
) $i \bar{i},{ }^{*}$-ieistì, -iei-t (avec à la II une accumulation de ${ }^{*}-e i+{ }^{*}-s$ et ${ }^{*}$-tha, par infixation de la désinence active, $\$ 1$, et à la III une recaractérisation active, comme celle du présent vén. ati-stei-t). Le type ${ }^{*}-\overline{0},{ }^{*}-e-s(i),{ }^{*}-e-t i$ (lat. ago, etc.) témoigne d'un pas de plus dans l'adéquation à la diathèse dynamique de l'actif morphologique : toute désinence moyenne a disparu de la II. Cette voie a été ouverte par la III plur. d'origine active (agunt, etc.), comme la personne correspondante des présents hittites en - $h i$; et ${ }^{*}-e-t i$, d'où ${ }^{*}-e-s(i)$, vient de la réfection de ${ }^{*}-e$ sur le modèle des actifs athématiques *-ti/-nti.

10.5. Le dernier transfert du moyen à l'actif ici retenu sera celui où le moyen a laissé sa marque au vocalisme radical, zéro, plus qu'à la flexion, où s'étend l'actif : ainsi de ${ }^{*} d e h_{3}$ - (dont le moyen fondamental ${ }^{*} d h_{3}-e$ subsiste, par-delà des recaractérisations flexionnelles actives, dans skr. á-da-t, lat. trā-di-t (si, de manière épidictique, on en fait un composé de ${ }^{*} d e h_{3^{-}}$, non de *dheh $\left.1^{-}\right)$, cf. fal. por-ded 3ème sg. perfectum, lat. de-ded, etc.), da-t. L'extension de la flexion active se fait plus largement en arménien (tam) qu'en latin $(d \bar{o})$ à la I.

10.6. Un paradigme comme lat. do, dat est mixte du point de vue des désinences ; et il n'existe aucun présent latin de morphologie entièrement active, puisque la I y est toujours en *-o, sauf dans sum, qui a le degré zéro du moyen, comme dat. Nombreux sont par ailleurs les paradigmes mixtes, qui témoignent de l'antériorité du moyen sur l'actif. On connaît bien le type fero, fer-t, où se sont rencontrés les deux vocalismes ${ }^{*}-e-$ : le ${ }^{*}-e-$ non alternant du présent autonome ancien, qui est celui des formes thématiques (ferō, ferimus, peut-être ferunt, ambigu), et le *-e-/zéro de l'actif (d'où fer-t, 
Françoise Bader

fer-s, et de là fer-tis) ; l'extension de l'actif est plus grande dans "aller", où du présent en *-e-ne subsiste plus que $e \bar{o}$ (cf. skr. áy $\bar{a}-(n i)$, subjonctif). Mais il existe de nombreux autres types de paradigmes mixtes, comme, à $l^{\prime}$ inverse de ${ }^{*}-\bar{\sigma} /-t,{ }^{*}-o m\left({ }^{*}-e m\right) /-e$, entrevu à propos de l'aoriste radical thématique, $\$$ 9.2., et qui est celui de tous les présents de l'agni. Parfois, la mixité est en même temps celle des vocalismes radicaux : hitt. uhhi / auszi "voir" a à la I une forme entièrement moyenne par son degré zéro et sa désinence, et à la III une forme entièrement active par son vocalisme *-eet sa désinence; même phénomène dans lat. s-um / es-t à l'égard des degrés radicaux, non de la flexion, puisque la désinence active s'est étendue à la I, dans ce seul verbe, dont la diathèse sémantique n'a pourtant rien de dynamique.

11. C'est que la flexion d'origine active a été, elle aussi, le siège d'un transfert, non plus à l'intérieur de la diathèse, mais de la diathèse au temps : au fur et à mesure que les formes d'origine moyenne devenaient récessives, hors des conjugaisons à double diathèse sémantique, pour être affectées, le plus souvent au prétérit (\$ 12.2.), les formes actives ont eu tendance à prendre un tropisme présent, dès avant l'emploi d'une particule temporalisante, comme le montre s-um, mais surtout après : l'opposition de diathèse entre un "être" et un "aller", dont les évolutions flexionnelles ont été largement parallèles, a en quelque sorte été neutralisée en faveur d'une catégorie commune, celle qui a commandé toute l'expansion de la conjugaison i.e., le temps, dont la forme axiale est le présent; d'où *es-mi "je suis" comme *ei-mi "je vais".

En effet, avec le développement de la catégorie de temps, le présent s'est sans cesse renouvelé, soit pour la I de "aller", de ${ }^{*} h_{1} i-h_{2} e(i)$ :

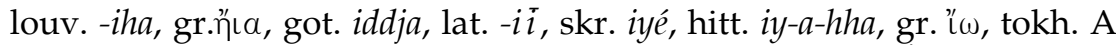

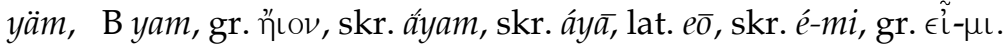

$\mathrm{Et}$, à chaque renouvellement, la forme antérieure dans le développement du moyen à l'actif a été rejetée soit vers l'éventuel (gr. "l $\omega$, skr. áya), soit, plus souvent, vers le prétérit, dans ses deux variétés flexionnelles successives : l'une, bien connue, à désinences "secondaires" actives (áyam, etc.), l'autre antérieure, à désinences d'origine moyenne (louv. -iha, etc.). C'est la dernière née de ces formes, du type skr. émi, qui est la plus fortement marquée à l'égard du présent, conférant ainsi à sa flexion une

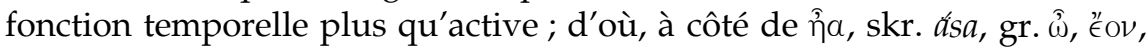
tokh. A na-sam, lat. sum, hitt. es-mi, etc.

12. Si le développement de la catégorie de temps a ainsi eu des conséquences pour l'érosion de la diathèse au profit du présent dans * $h_{1}$ es-mi, le développement d'un actif opposé au moyen par adéquation à la diathèse sémantique dynamique d'une voix morphologique propre a entraîné pour l'ancien moyen une scission en moyen sémantique (s'opposant à un actif 
dans une conjugaison à double diathèse, avec les valeurs données par B., cf. § 4.1.), et moyen résiduel ayant perdu toute valeur de diathèse, par récession, et non par expansion comme dans le cas de l'actif morphologique "je suis".

12.1. Le moyen résiduel est parfois un impersonnel : c'est le cas de lat. ìtur, à degré radical ambigu: ${ }^{*} h_{1}$ ei- comme dans is, it? ou plutôt zéro, par contraction en $\dot{i}-$, devant consonne, de la géminée née du traitement de ${ }^{*} h_{1} i->{ }^{*} i h_{1}$ - (métathèse) $>i y-$, par assimilation, subsistant devant voyelle (thématique) dans hitt. iy-a-tta-ri ? cf., athématique, hitt. paitta, prétérit de paimi (III plur. päir, cf. lat. -iēre).

12.2. Plus souvent, le moyen récessif est prétérital. Il peut être employé dans deux séries de formes, les unes radicales, à degré zéro, provenant de l'ancien moyen fondamental, comme $\ddot{\eta} \iota, i i$, etc., les autres, affixées,

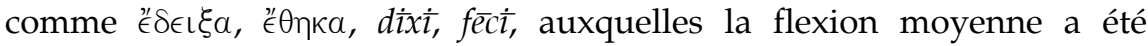
étendue, à partir de ces formes radicales, avec sa seule valeur temporelle (et la "confusion du parfait et de l'aoriste" en latin ne vient que de l'identité originelle de flexion entre le moyen fondamental devenu prétérit $(i \bar{\imath}, f u \hat{\imath})$, le parfait intégré lui aussi au prétérit (actif) des deux types uìd $\dot{\imath}$, dedì, l'aoriste affixé à flexion d'origine moyenne, dìxì). Une fois de plus, la désinence de III sg. peut être à côté de ${ }^{*}-e,{ }^{*}$-to, également dans des prétérits radicaux et affixés ; ainsi, pour ces derniers, vén. dona.s.to (à côté de la III plur. donasa.n. active comme aux présents en -hi, aux présents thématiques, et au parfait grec), hitt. tamasta (III plur. tamassir), cf. gr. $\delta a ́ \mu a \sigma \sigma \epsilon$ et $\delta a \mu a ́ \sigma \sigma a \tau o$. Les formes radicales sont vivantes en hittite où les présents en -mi comme es-zi ont un prétérit comme es-ta; ce type est

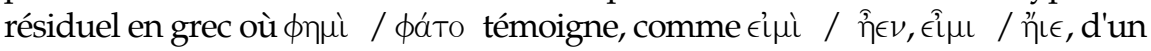
transfert à la catégorie de temps de l'opposition morphologique entre Actif et Moyen dans le verbe. 
Françoise Bader

\section{Bibliographie}

\section{Travaux d'E. Benveniste cités}

1931 : Trois étymologies latines [aprïlis, dēns, nemus]. BSL 32, 68-85

1933 : L'anaphorique prussien din et le système des démonstratifs indo-européens. Studi Baltici 3, 121-130

1935 : Origines de la formation des noms en indo-européen. Paris : Maisonneuve

1936: Tokharien et Indo-Européen, in: Germanen und Indogermanen. Festschrift für H.Hirt. Heidelberg : C.Winter, 90-109

1946 : Structure des relations de personne dans le verbe. BSL 43 (1946, publié en 1947), 1-12 = PLG I, 225-236

1949 : Sur quelques développements du parfait indo-européen. Archivum linguisticum 1, 16-22

1950 : ACTIF ET MOYEN DANS LE VERBE. JPsych. 43, 121-129 = PLG I, 168-175 (cité ici dans la pagination des PLG)

1950 b : La phrase nominale. BSL 46 = PLG I, 151-167

1951 : Don et échange dans le vocabulaire indo-européen. L'Année sociologique. 3ème série, 2,7-20 = PLG I, 315-326

1952 : La construction passive du parfait transitif. BSL 48,52-62 = PLG I, 176-186

1955 : Présents dénominatifs en hittite, in : Corolla linguistica. Festschrift F. Sommer. Wiesbaden : Harrassowitz, 1-4 = 1962, 20-26 (avec additions et corrections)

$1959 a$ : Les relations de temps dans le verbe français. BSL 54, 69-82 = PLG I, 237-250

$1959 b$ : Sur la phonétique et la syntaxe de l'arménien classique. I. La mutation consonantique et les dialectes modernes. II. Transitif et intransitif dans la construction du parfait. BSL 54, $46-68$

1960 : "Etre" et "Avoir" dans leurs fonctions linguistiques. BSL 55 = PLG I, 187-207

1962 : Hittite et Indo-Européen. Etudes comparatives. Paris : Maisonneuve

1966 : Problèmes de linguistique générale I. Paris : Gallimard

$1970 a$ : L'appareil formel de l'énonciation. Langages 17, 12-18 = PLG II, 79-88

$1970 b$ : Définition d'un parfait en paléo-sibérien oriental, in : Studies in general and oriental Linguistics presented to Shiro Hattori..., Tokio, 6-9

1974 : Problèmes de linguistique générale II. Paris : Gallimard

\section{Autres.}

A. J'ai cité (ici donnés dans l'ordre chronologique) :

1928 : RENOU, Louis, Les formes dites d'injonctif dans le Rgveda, in : Etrennes de linguistique offertes par quelques amis à Emile Benveniste. Avant-Propos de A.Meillet. Paris : P.Geuthner, 63-80

1967 : HOFFMANN, Karl, Der Injunktiv im Veda. Heidelberg : C.Winter

1969: WATKINS, Calvert, Indogermanische Grammatik III/I : Geschichte der indogermanischen Verbalflexion. Heidelberg : C.Winter

1984 : FLOBERT, Pierre : Benveniste et le problème de la diathèse, in : E.Benveniste aujourd'hui. Paris : Société pour l'information grammaticale, 51-61 (je lui dois l'opposition entre voix morphologique et diathèse sémantique)

B. La synthèse ici présentée a été amorcée par mes travaux antérieurs, sur le verbe et sur le pronom :

$1^{\circ}$ verbe

a) diathèse (outre de nombreux rapports de l'Annuaire E.P.H.E. IV) :

1984 : Autour de Polyphème le Cyclope à l'oeil brillant : diathèse et vision. Die Sprache 30/2, 17-49 
b) temps :

1974 : Persée, perthōet l'expression archaïque du temps en indo-européen. BSL 69, 1-53

c) parfait :

1967 : Le système des désinences de 3ème personne du pluriel du perfectum latin. BSL 62,86-105

$1968 a$ : Relations de structure entre les désinences d'infectum et de perfectum en latin. Word 24, 14-47

$1968 b$ : Vocalisme et redoublement au parfait radical latin. BSL 63, 160-196

1969 : Eikôs, eoikôs et le parfait redoublé en grec. BSL 64, 57-100

1972 : Parfait et moyen en grec, in : Mélanges de linguistique et de philologie grecques offerts à P.Chantraine. Paris : Klincksieck, 1-21

d) présents :

$1975 a$ : Vieil irlandais no, no- et les formes tokhariennes apparentées. EC 14, 391-403

$1975 b$ : La structure thématique aux présents hittites. RHA 33, 5-29

1976 : Le présent du verbe "être" en indo-européen. BSL 71, 27-111

1979 : Les présents à nasale indo-européens : la classe en *-nu-. BSL 74, 191-235

1980 : Présents moyens hittites à vocalisme *-e- et formations de présents indo-européens, in : Recherches de linguistique. Hommage à M.Leroy. Bruxelles, 21-40

1984-85 : Annuaire E.P.H.E. IV, 146, sur le type iungō

1987 : Hittite duratives and the problem of IE present-formations with infix and suffix. JIES 15, 131-156

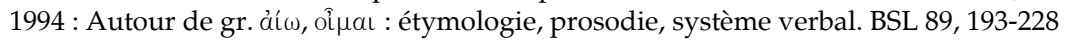

e) aoriste :

1978 : Flexions d'aoristes sigmatiques, in : Etrennes de Septantaine. Travaux de linguistique et de grammaire comparée offerts à Michel Lejeune. Paris : Klincksieck, 29-44

f) pour la racine *kel-, ici évoquée $\$ 7.3$. :

1983 : De l"'auscultation" à la "célébrité". Formes de la racine *kel-, in : Hommages à Jean Cousin. Rencontres avec l'Antiquité classique. Paris-Besançon, 27-60 (pour le *wei-d- du § 8.2., voir a) diathèse : 1984, 17-49)

$2^{\circ}$ pronom : ce sont les agglutinations au verbe des pronoms qui ont été au point de départ de mes recherches sur ceux-ci. Je m'y étais consacrée pour le précédent colloque consacré à Benveniste :

1984 : Benveniste et les pronoms, in : E. Benveniste aujourd'hui. Actes du Colloque international du CNRS. Tours, 1983. Paris : Société pour l'Information grammaticale, 17-49.

Depuis, j'ai publié en ce domaine notamment:

1986 : Structure de l'énoncé indo-européen. BSL 81, 71-120

1989-90 (1991) : Comparaison typologique de l'étrusque et de l'indo-européen : structures agglutinantes. Studi Etruschi 56, 155-173 (156-164 pour les problèmes ici abordés) ;

et trois articles de synthèse :

1990 : Les pronoms dans les langues indo-européennes. MSL, N.S. 1, 23-35

1992 : Formes, fonctions, étymologies pronominales, in : La Deixis, sous la direction de M.A.Morel et L.Danon-Boileau. Paris : PUF, 27-41

1994: Fonctions, catégories et sémantisme des pronoms, in: Florilegium Historiographiae linguisticae. Etudes d'historiographie de la linguistique et de la grammaire comparée à la mémoire de Maurice Leroy. Louvain-la-Neuve : Peteers, 29-78, spécialement 33-40 pour le "supplétisme verbo-pronominal". 